\title{
A BENCHMARKING OF SIX SOFTWARE PACKAGES FOR FULL-WAVE ANALYSIS OF MICROSTRIP ANTENNAS
}

\author{
A. Vasylchenko* ${ }^{\star}$, Y. Schols ${ }^{*}$, W. De Raedt ${ }^{\dagger}$, G. A. E. Vandenbosch* \\ *Katholieke Universiteit Leuven, ESAT-TELEMIC, \\ B-3001 Leuven, Belgium \\ alexander.vasylchenko@esat.kuleuven.be; \\ ${ }^{\dagger}$ MMEC, B-3001 Leuven, Belgium
}

Keywords: Benchmarking, planar antennas, electromagnetic simulation, full-wave solvers

\begin{abstract}
An explicit quality assessment of full-wave EM solvers for planar antennas is presented, where the solvers are introduced and categorized on the basis of their solution method: Integral Equations (IE), Finite Elements (FE) or Finite Integration Technique (FIT). Based on their theoretical specificities, each method is linked to a certain structure profile. All EM solvers were initially compared by analysing a classical square patch on an homogeneous substrate. By introducing dielectric inhomogeneity in the second antenna the solvers were confronted with a greater challenge to EM modelling. Possible mutual differences of the simulations results are interpreted and validated with microstrip antenna measurements.
\end{abstract}

\section{Introduction}

Nowadays, antenna engineers can rely on highly specialized full-wave electromagnetic (EM) field solvers to develop and optimize their designs. The operation of these solvers is based on the numerical solution of Maxwell equations in differential or integral form. It is evident that the underlying solution method significantly influences the efficiency and accuracy with which certain structure types are analyzed. Nevertheless, the commercial focus increasingly switches from such key theoretical considerations to improvements in the area of layout tools and system-level design tools. Therefore, users may get the wrong impression that a given solver is automatically suited to solve any kind of problem with arbitrary precision. This paper verifies the plausibility of such expectations by presenting an extensive benchmark study which focuses on the capabilities and limitations of the applied EM modelling theories that usually remain hidden for the antenna designer.

The benchmarking of six electromagnetic simulation programs was carried out on the produced antennas. These programs are introduced in different categories, based on: Method of Moments (MoM), Finite Elements Method (FEM) and Finite Integration Technique (FIT). The simulation results are mutually compared and set against the measurement results. It is important to note that only theoretical considerations will be taken into account: the benchmark study does not include the comparison of Graphical User Interfaces (GUI), price/performance ratio, user friendliness, and such like. In conclusion, a qualitative interpretation from a theoretical point of view for quantitative differences is given. Finally, some general guiding rules are formulated.

\section{Introduction to examined full-wave solvers}

Because the 3D field solver MAGMAS 3D, developed at K.U.Leuven, is E-based and initially, it was obviously tested by comparison with other IE commercial simulators. Since commercial solvers are often very expensive, their availability is limited. Apart from K.U.Leuven MAGMAS 3D, other affordable $\mathbb{E}$ solvers are included in this benchmark, namely, E3D of Zeland Software, FEKO of EM Software \& Systems and ADS Momentum of Agilent. Two of the leading differential EM tools: HFSS of Ansoft for the FE method and CST Microwave Studio (CST MWS) for the FIT/FEM methods were also introduced for the sake of objectivity. Because the qualitative performance of a field solver largely depends on the underlying numerical method, the different solvers are now discussed.

- ADS Momentum [1]: The integral equations are formulated in mixed potential form and the matrix elements are evaluated completely in the spatial domain. Momentum was originally developed to analyze planar circuitry. Per layer, the vertical currents are assumed constant, which limits the modeling capabilities for full $3 \mathrm{D}$ structures. In the same way, the modeling of finite dielectric volumes is not included.

- MAGMAS 3D [9], [11]: The matrix elements are computed using a hybrid dyadic-mixed potential formulation and a combined spectral-space domain approach. A lot of analytical work is included in the spectral domain which makes the code computationally efficient. Surface and volume currents are decomposed in horizontal and vertical currents (quasi 3D approximation), which are both expanded using generalized rooftop functions.

- $\quad$ E3D [14]: The integral equations are formulated with a full dyadic Green's function and the matrix elements are computed completely numerical in the spatial domain. 
IE3D can model truly arbitrary 3D metal structures; since 2006 also finite $3 \mathrm{D}$ dielectric volumes can be modeled with a Volume Integral Equations (VIE) approach.

- FEKO [5]: The matrix elements are computed using a mixed-potential formulation and a spatial domain approach. The solver can model truly arbitrary 3D structures. Dielectric volumes can be modeled in three different ways: Surface Integral Equations (SE) approach, VIE approach and a hybrid approach with the FE method

- HFSS [2]: automatically divides the geometric model into a large number of tetrahedra, where a single tetrahedron is a four-sided pyramid. This collection of tetrahedra is referred to as the finite element mesh. Each element can contain a different material. Therefore, the interface between two different materials must coincide with element boundaries [8]. The value of a vector field quantity (such as the $\mathrm{H}$-field or E-field) at points inside each tetrahedron is interpolated from the vertices of the tetrahedron. The field inside each tetrahedron is interpolated from these nodal values. By representing field quantities in this way, the system can transform Maxwell's equations into matrix equations that are solved using traditional numerical methods.

- CST MWS [4] is based on the finite integration technique (FIT) first proposed by Weinland in 1976 [12] and allows to choose the time domain as well as the frequency domain approach. Despite the variety between transient, eigenmode, and frequency domain solvers within CST MWS, the transient solver was examined for benchmarking as the flag ship module of CST MWS. The transient CST MWS solver is a popular time domain approach represented among commercially available software packages. The Time Domain Solver calculates the broadband behavior of electromagnetic devices in one simulation run with an arbitrarily fine frequency resolution, and without missing any resonance peaks. In the case of Cartesian grids the FIT formulation can be written in the time domain to yield standard FDTD methods.

\section{Test designs physical realization}

Two antennas were selected for further investigation. These antennas have a vast number of applications and thus, their accurate modeling has become an important issue. In [10] it is shown how the radiation pattern of patch antennas on a dielectric substrate is improved considerably when the dielectric material under the patch is locally removed, while in [13] the same technique is applied to control the surface wave coupling with other radiating elements mounted on the same substrate. In addition to their great potential, antennas on locally modified or inhomogeneous substrates are also interesting from a modeling point of view. They combine both finite and infinite dielectric regions in the same configuration and thus form a true challenge for both differential and integral equation modeling methods. As long as the dielectric inhomogeneities remain small or localized, they can be described efficiently by quasi-3D volume currents, while the extended dielectric substrate can be contained in the multilayered Green's functions.

\subsection{Test antennas design}

The antennas sketch is depicted in Fig. 1. A square resonant patch of dimensions $15.2 \times 15.2 \mathrm{~mm}^{2}$ is situated on a one side cladded, 60 mil thick Rogers RO4003 substrate, characterized by a permittivity of $\varepsilon_{R 04003}-(3.38-j 0.0074) \varepsilon_{0}$. The patch is fed by a $3.8 \mathrm{~mm}$ wide microstrip line. The idea is now to investigate how the operation of this antenna changes when the substrate material under the patch is locally removed and replaced by a Rogers RO3003 substrate with permittivity $\varepsilon_{\mathrm{R} 03003}-(3.00-\mathrm{j} 0.0039) \varepsilon_{0}$. We therefore analyze this antenna for a completely homogeneous substrate, i.e. without volume and $\varepsilon_{d}-\varepsilon_{\mathrm{RO} 4003}$, and compare this with the results for a second simulation setup, including a $15.2 \times 15.2 \times 1.52$ $\mathrm{mm}^{3}$ dielectric volume of $\varepsilon_{\mathrm{d}}-\varepsilon_{\mathrm{RO} 3003}$ under the patch.

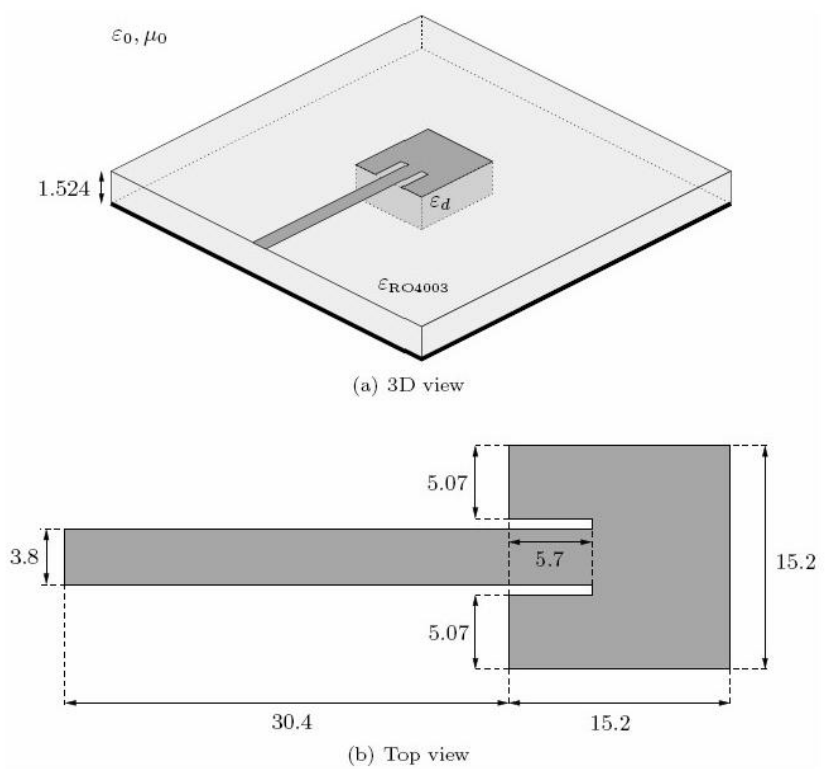

Figure 1: Line-fed patch antenna on an extended Rogers RO4003 substrate, containing a small dielectric volume of permittivity $\varepsilon_{\mathrm{d}}$ under the patch. Dimensions are given in $\mathrm{mm}$.

\subsection{Antennas realization and measurement setup}

To be able to look at the simulation results in a wider perspective, the two antenna designs were effectively realized and measured (Fig. 2). The homogeneous antenna, depicted on Fig. 3, was realized pragmatically by mechanically etching the required metallic shape on the RO4003 substrate. The fabrication of the inhomogeneous design was somewhat more involved. The feeding line was etched on the RO4003 substrate with a square hole spared out at the position of the patch. An equally sized piece of RO3003 substrate, with only the insets of the patch etched out, was then fitted in the hole to construct the antenna. The electrical conductivity was ensured by soldering the metal junctions. The strip lines are fed by a straight coaxial PCB connector of Huber \& Suhner: type SMA-S50-0-45, mounted on the edge of the substrate. 


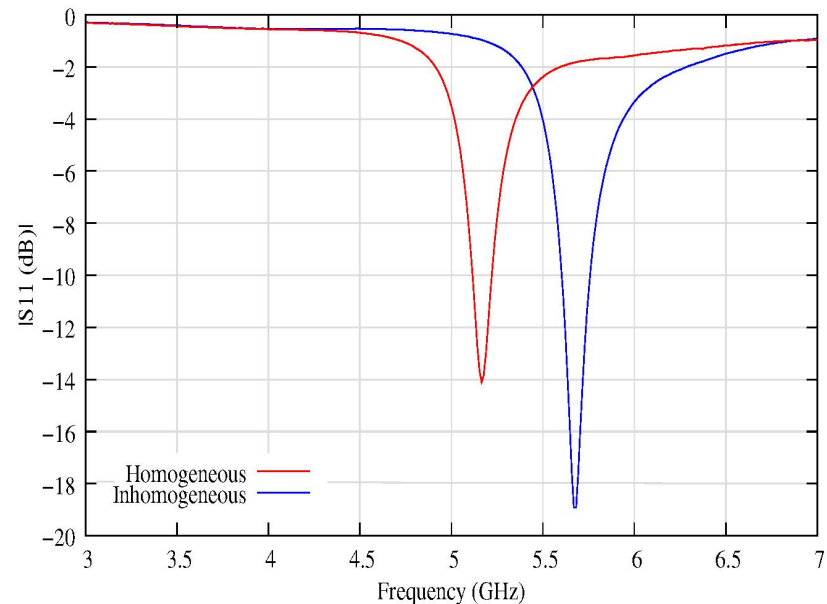

Figure 2: Measured amplitude of the $\mathrm{S} 11$ - parameter for the realized line-fed patch antennas.

In the measurements a $50 \Omega$ SMA is connected to the end of the feeding strip and grounded to the edge of the ground plane. An RF cable from the Vector Network Analyzer (HP85107B) is connected to the SMA to excite every Antenna Under Test (AUT). The measurements of small antennas in an anechoic chamber are very sensitive to the presence of the RF cable located in the near field of the AUT [3]. The additional radiation from the outer surface of the signal cable has a significant impact on measurement parameters such as operating frequency, radiation pattern, and gain. Ferrite chokes around the front end of the cable were used to reduce the additional radiation from the signal cable by absorbing the energy on the outer surface of the cable [7].

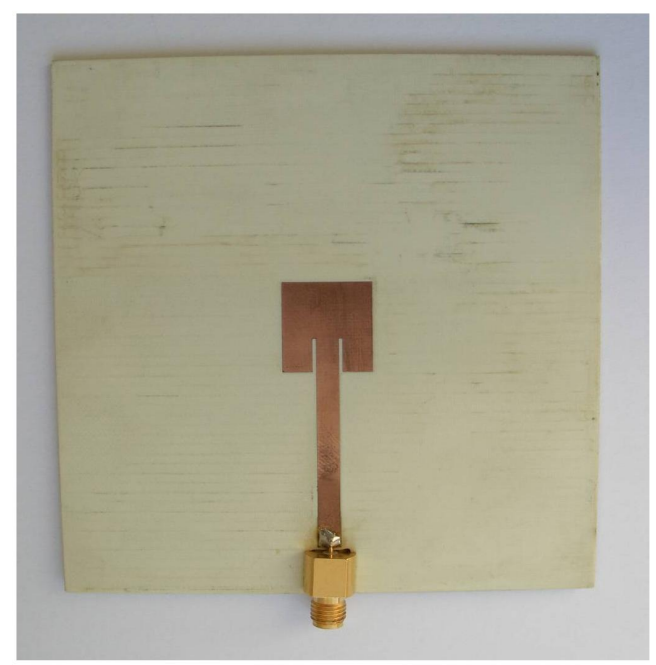

Figure 3: Top view of the analyzed line-fed patch on the homogeneous substrate with SMA connector

\section{Benchmarking overview}

The simulation results in Fig. 4 and 5, show the amplitude of the $S_{11}$ parameter from 4.5 to $6.5 \mathrm{GHz}$. Tables 1 and 2 signify the data in Fig. 4 and 5 in absolute values. The minimal and maximal frequencies crossing the Return Loss level at $-10 \mathrm{~dB}$ are Fmin and Fmax respectively. The difference between Fmin and Fmax defines the bandwidth (BW) at S11 $=-10 \mathrm{~dB}$.

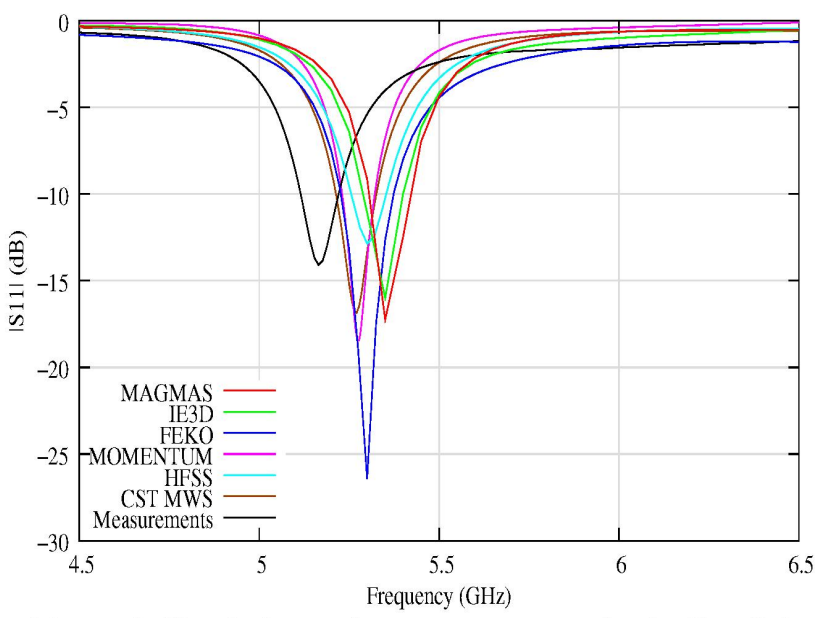

Figure 4: Simulation and measurement results for line-fed patch antenna on an homogeneous substrate.

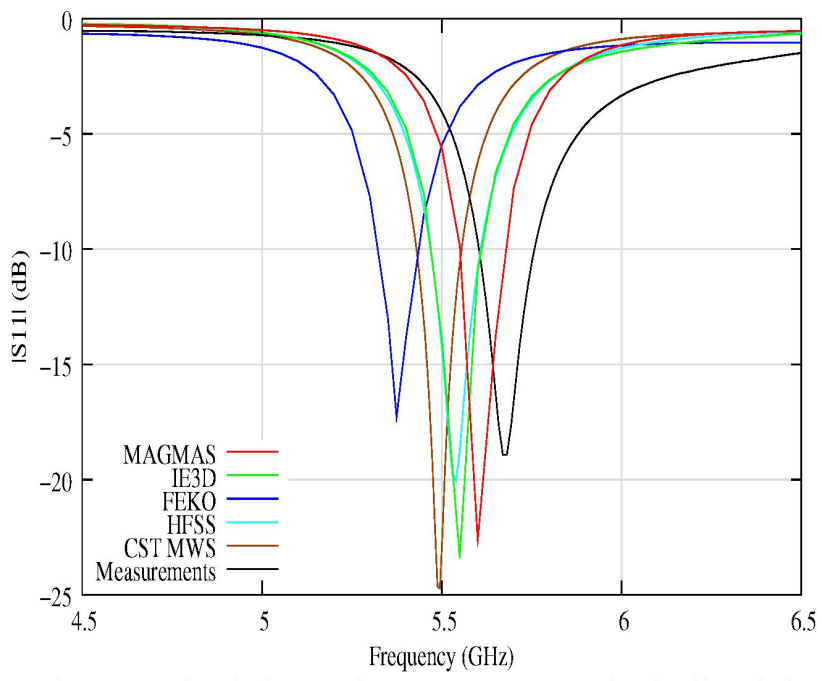

Figure 5: Simulation and measurement results for line-fed patch antenna on an inhomogeneous substrate.

The simulation results predict a comparable shift to a higher resonance frequency, when the RO4003 substrate is locally replaced by the RO3003 substrate. This agrees with the fact that material of lower dielectric density is added to the structure, such that the overall antenna becomes electrically smaller. This is also confirmed by the measurement results in Fig. 2. Closer investigation of the simulation results reveals that the mutual correspondence between the different solvers is certainly not perfect. In order to comprehend such a difference, the central frequency $(\mathrm{Fc})$ is determined by $\mathrm{Fc}=$ Fmax - Fmin. Hence, Fc-shift is the magnitudes deviation between $\mathrm{Fc}$ measured and $\mathrm{Fc}$ simulated. The simulated central frequency deviation $(\mathrm{FcD})$ has a purpose to exclude fabrication and consequently measurement inaccuracies. The 
central frequency deviation $(\mathrm{FcD})$ shows dispertion about the mean of simulated results only. It is defined as $\mathrm{FcD}=\mathrm{Fc}-$ FcAV, where

$$
F c A V=\frac{1}{n} \sum_{i=1}^{n} F c
$$

The FcD and FcAV parameters are summarized for both antennas in Table 3.

\begin{tabular}{|c|c|c|c|c|}
\hline $\begin{array}{c}\text { Software } \\
\text { Package }\end{array}$ & $\begin{array}{c}\text { Fmin } \\
(\mathbf{G H z})\end{array}$ & $\begin{array}{c}\text { Fmax } \\
(\mathrm{GHz})\end{array}$ & $\begin{array}{c}\text { Fc } \\
(\mathbf{G H z})\end{array}$ & $\begin{array}{c}\text { BW } \\
(\mathbf{G H z})\end{array}$ \\
\hline MAGMAS & 5.305 & 5.423 & 5.364 & 0.118 \\
\hline IE3D & 5.29 & 5.4 & 5.345 & 0.11 \\
\hline FEKO & 5.226 & 5.374 & 5.3 & 0.148 \\
\hline MOMENTUM & 5.23 & 5.322 & 5.276 & 0.092 \\
\hline HFSS & 5.255 & 5.353 & 5.304 & 0.098 \\
\hline CST MWS & 5.213 & 5.326 & 5.27 & 0.113 \\
\hline Measurement & 5.115 & 5.22 & 5.168 & 0.105 \\
\hline
\end{tabular}

Table 1: Nominal frequency values for patch antenna on an homogeneous substrate at Return Loss level of $-10 \mathrm{~dB}$

\begin{tabular}{|c|c|c|c|c|}
\hline $\begin{array}{c}\text { Software } \\
\text { Package }\end{array}$ & $\begin{array}{c}\text { Fmin } \\
(\mathbf{G H z})\end{array}$ & $\begin{array}{c}\text { Fmax } \\
(\mathbf{G H z})\end{array}$ & $\begin{array}{c}\text { Fc } \\
(\mathbf{G H z})\end{array}$ & $\begin{array}{c}\text { BW } \\
(\mathrm{GHz})\end{array}$ \\
\hline MAGMAS & 5.55 & 5.68 & 5.615 & 0.13 \\
\hline IE3D & 5.47 & 5.611 & 5.541 & 0.141 \\
\hline FEKO & 5.322 & 5.437 & 5.38 & 0.115 \\
\hline MOMENTUM & NA & NA & NA & NA \\
\hline HFSS & 5.47 & 5.608 & 5.539 & 0.138 \\
\hline CST MWS & 5.43 & 5.555 & 5.493 & 0.125 \\
\hline Measurement & 5.603 & 5.758 & 5.681 & 0.155 \\
\hline
\end{tabular}

Table 2: Nominal frequency values for patch antenna on an inhomogeneous substrate at Return Loss level of $-10 \mathrm{~dB}$

\begin{tabular}{|c|c|c||c|c|}
\hline \multirow{2}{*}{$\begin{array}{c}\text { Software } \\
\text { Package }\end{array}$} & \multicolumn{2}{|c|}{$\begin{array}{c}\text { Homogeneous } \\
\text { Substrate }\end{array}$} & $\begin{array}{c}\text { Inhomogeneous } \\
\text { Substrate }\end{array}$ \\
\cline { 2 - 5 } & $\begin{array}{c}\text { Fc-shift } \\
(\%)\end{array}$ & $\begin{array}{c}\text { FcD } \\
(\%)\end{array}$ & $\begin{array}{c}\text { Fc-shift } \\
(\%)\end{array}$ & $\begin{array}{c}\text { FcD } \\
(\%)\end{array}$ \\
\hline MAGMAS & 0.038 & 1.02 & 0.012 & 1.84 \\
\hline IE3D & 0.034 & 0.66 & 0.025 & 0.50 \\
\hline FEKO & 0.026 & -0.19 & 0.053 & -2.42 \\
\hline MOMENTUM & 0.021 & -0.64 & NA & NA \\
\hline HFSS & 0.026 & -0.11 & 0.025 & 0.46 \\
\hline CST MWS & 0.02 & -0.75 & 0.033 & -0.37 \\
\hline Measurement & 0 & -2.67 & 0 & 3.04 \\
\hline
\end{tabular}

Table 3: Relative frequency values for the both antennas (FcD value is derived from the simulation results only)

The accuracy for the simulated center frequencies is comparable for all solvers and is generally better than $5 \%$. Possible explanations for the observed deviations are the differences in feed model used and imperfections in the realizations. Namely, coax-to-microstrip transmission realized by soldering a 500hm SMA connector causes the most significant differences between the antenna models and their practical realization. The connector characterization with its following de-embedding from the measurements results has to be executed [6]. By considering $\mathrm{FcD}$ value the simulation results have a relatively small dispersion. The deviation within $1.77 \%$ for an antenna on an homogeneous substrate shows a good agreement for all software packages, whereas the more complex design on an inhomogeneous substrate exposes $4.26 \%$ of $\mathrm{FcD}$ discrepancy.

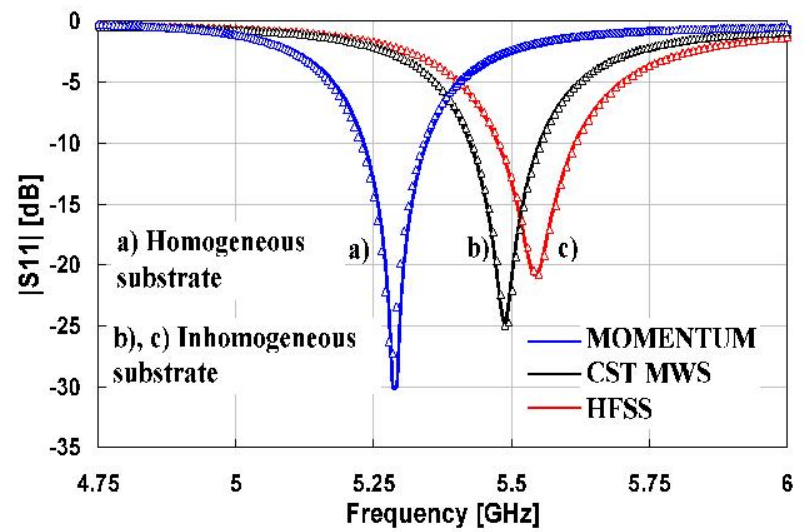

Figure 6: Convergence of the simulation results for line-fed patch antenna.

A comparative convergence study of three EM solvers is demonstrated in Fig. 6. Forcing HFSS to double the amount tetrahedra for antenna model discretization or number of mesh cells per wavelength in Momentum and CST MWS (triangular markers) do not affect the solution significantly, if a convergence criterion of the method is met. It is noticeable, that the major results deviation between different solvers is mostly defined by use of the different modeling techniques on which every EM solver is based. Every software package provides sufficiently accurate solution, even if default simulation options are applied.

Comparative simulation time in seconds per frequency point (SPFP) as well as number of unknowns employed per structure is represented in Table 4. Number of unknown refers to EM problem sizes when the method convergence is reached. SPFP gives an opportunity to determine a possible difference in calculation efficiency between the solvers. The simulations are executed on Intel Xeon ${ }^{\mathrm{TM}} 2.80 \mathrm{GHz}$ Pentium 4 processor Slackware Linux using different operating systems: Slackware Linux 10.0 for the MAGMAS simulation and Windows NT for the $\mathbb{E} 3 \mathrm{D}$ and CST MWS simulations. FEKO was run on AMD Athlon ${ }^{\mathrm{TM}} 643800+$ processor operated by Linux x86 64 2.6.11.9. The 2 x Dual-Core AMD Opteron ${ }^{\mathrm{TM}} 285(2.6 \mathrm{G} \overline{\mathrm{H} z}) 64$ bit with Windows Server 2003 and $2 \times$ AMD Opteron ${ }^{\mathrm{TM}} 250(2.4 \mathrm{GHz}) 64$ bit with Red Hat Linux 2.4.21 were used for HFSS and Momentum simulations respectively. For both antennas a symmetry plane was applied in HFSS and CST software packages. If the symmetry plane 
is specified, the calculation domain is cut by half. This reducing the size of the problems helps speed up the solution time.

\begin{tabular}{|c|c|c|c|c|}
\hline \multirow{2}{*}{$\begin{array}{l}\begin{array}{c}\text { Antenna } \\
\text { substrate }\end{array} \\
\begin{array}{l}\text { Software } \\
\text { package }\end{array}\end{array}$} & \multicolumn{2}{|c|}{ Homogeneous } & \multicolumn{2}{|c|}{ Inhomogeneous } \\
\hline & 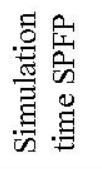 & 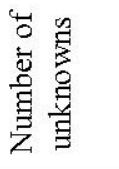 & 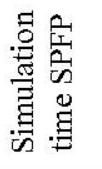 & 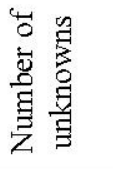 \\
\hline MAGMAS & 38 & 1275 & 1219 & 5403 \\
\hline IE3D & 39 & 2223 & 4757 & 9396 \\
\hline FEKO & 70 & 1424 & 1151 & 10113 \\
\hline Momentum & 2 & 384 & NA & NA \\
\hline CST MWS & $105 *$ & 313728 & $188^{*}$ & 462672 \\
\hline HFSS & 23 & 60719 & 27 & 73395 \\
\hline
\end{tabular}

Table 4: Comparative simulation time for different software packages (* - total simulation time for a full frequency span)

\section{Conclusion}

Planar antennas offer many advantages such as a small size, a light weight, easy fabrication and they are suitable for the integration with other planar RF circuits. Due to the sophisticated requirements of microwave applications, the planar antenna design has become a critical issue in modern system design. Therefore, considerable effort has gone into creating powerful programs for antenna simulation. These programs are based on different electromagnetic simulation techniques, allowing to model different antenna types, at the same time providing the end-user with an intuitive and understandable interface. From the study four important points have been observed:

- The simple square patch antennas can be predicted by every simulation program with results deviation not beyond $1.8 \%$. Moreover, while having a more attractive price, MoM based programs perform the simulations faster.

- The more challenging design like patch antenna on inhomogeneous substrate require finite substrate effects taken into account. Thus, FEM and FIT-based programs can show better agreement with the measurement, compared to programs using MoM. However, FEM and FIT are more "brute force" techniques, they generally require the inversion of very large matrices. The implementation of the complicated numerical methods makes FEM and FIT based programs memory intensive and expensive.

- A proper mesh generation and feeding model selection are two important issues predetermining the successful simulation in the software packages reviewed.
This parametric study has addressed the important role of the initial software selection for the variable antenna designs, and provided the practical guidance for the antenna engineers through comparison of popular theoretical methods. However, confronting major results deviation between different solvers with antenna measurements clearly shows that results diversity is mostly defined by exploiting different modeling techniques, but not by different solution setup capabilities within one EM solver. Therefore, by making a trade-off, we suggest that using at least two software packages based on different theoretical methods can minimize the discrepancy magnitude between the simulated and measured antenna characteristics.

\section{Acknowledgements}

The authors gratefully acknowledge Dr. Jian-X. Zheng from Zeland Software, Inc., Mr. David Prestaux and Mr. Alain Michel Ansoft France, Mr. Niels Berger EM Software \& Systems GmbH and Dr. Tilmann Wittig from CST GmbH for the willingness to share their EM solvers expertise.

\section{References}

[1] Agilent Technologies, EEsof EDA, Momentum, http://eesof.tm.agilent.com/products/momentum_main. html.

[2] Ansoft Corporation, HFSS v. 10.1.1 user manual, Jul 2006, http://www.ansoft.com.

[3] Z. N. Chen, N. Yang, Y. X. Guo, and M. Y. W. Chia, "An investigation into measurement of handset antennas," IEEE Trans. Instrum. Meas., vol. 54, no. 3, pp. 1100-1110, June 2005.

[4] CST GmbH., CST Microwave Studio v. 2006B, www.cts.com.

[5] EMSS - EM Software \& Systems Ltd, FEKO Suite 5.2 user manual, jan 2006, http://www.emss.co.za.

[6] M. A. Goodberlet and J. B. Mead, "Microwave Connector Characterization," IEEE Microwave Magazine, vol. 7, no. 5, pp. 78-83, Oct. 2006.

[7] C. Icheln J. Ollikainen and P. Vainikainen, 'Reducing the influence of feed cables on small antenna measurements," Electron. Lett., vol. 35, no. 15, pp. 1212-1214, Jul. 1999.

[8] N. Ida, Engineering Electromagnetics, Springer, 2003.

[9] MAGMAS 3D, K.U.Leuven ESAT-TELEMAC, http://www.esat.kuleuven.be/telemic/antennas/magmas

[10] M. J. Vaughan, K. Y. Hur, and R. C. Compton, "Improvement of microstrip patch antenna radiation 
patterns," IEEE Trans. Antennas Propagat., vol. 42, no. 6, pp. 882-885, June 1994.

[11] M. Vrancken and G.A.E. Vandenbosch, Hybrid dyadic-mixed-potential and combined spectral-space domain integral-equation analysis of quasi-3-D structures in stratified media, IEEE Trans Microwave Theory Tech, vol 51, 2003, pp. 216-225.

[12] T. Weiland, "A discretization method for the solution of Maxwell's equations for six-component fields", Electronics and Communications $A E \ddot{U}$, Vol. 31, No. 3, 116-120, 1977.

[13] J.-G. Yook and L. P. B. Katehi, "Micromachined microstrip patch antenna with controlled mutual coupling and surface waves," IEEE Trans. Antennas Propagat., vol. 49, no. 9, pp. 1282-1289, Sept. 2001.

[14] Zeland Software, Inc., IE3D v. 11.2 user manual, jan 2006, http://www.zeland.com. 